\title{
Modelado Cinético de la Disolución de Hidroboracita en Soluciones de Ácido Nítrico
}

\author{
Graciela V. Morales y Oscar D. Quiroga \\ Facultad de Ingeniería, Consejo de Investigación, INIQUI-CONICET, Universidad Nacional de \\ Salta, Avenida Bolivia 5150, 4400 Salta-Argentina (e-mail: gmorales@unsa.edu.ar)
}

Recibido Oct. 18, 2011; Aceptado Nov. 30, 2011; Versión final recibida May. 08, 2012

\begin{abstract}
Resumen
Se estudia la cinética de disolución de hidroboracita en soluciones de ácido nítrico, cuyos subproductos, nitrato de calcio y nitrato de magnesio, tienen una importante aplicabilidad comercial como fertilizantes. La disolución se realiza en un reactor tanque agitado discontinuo a presión atmosférica. Se investigan los efectos que tienen los factores temperatura, tamaño de partícula, relación sólido/líquido y concentración de ácido, sobre la velocidad de disolución; y se evalúan los parámetros cinéticos: factor de frecuencia y energía de activación. Se observa que la velocidad de disolución crece con el aumento de la temperatura, la disminución del tamaño de partícula, y con la disminución de la relación sólido/líquido. Se observa, también, que un aumento en la concentración del ácido nítrico hace aumentar, siempre, el grado de disolución. Se determina que la energía de activación aparente es del orden de $13 \mathrm{~kJ} / \mathrm{mol}$, siendo la disolución de la hidroboracita en ácido nítrico controlada por mecanismos difusivos.
\end{abstract}

Palabras clave: hidroboracita, ácido nítrico, disolución, cinética, modelado

\section{Kinetics Modeling of Hydroboracite Dissolution in Nitric Acid Solutions}

\begin{abstract}
The dissolution kinetics of hydroboracite in nitric acid solutions, whose products, calcium nitrate and magnesium nitrate have important commercial applications as fertilizer was studied. The dissolution was carried out in a batch reactor at atmospheric pressure. The effects of the temperature, particle size, solid/liquid ratio and concentration of solution on the dissolution rate were investigated, and the kinetic parameters: frequency factor and activation energy were evaluated. It was found that the dissolution rate increased when the reaction temperature increased and when the particle size decreases. The dissolution rate decreases when the solid/liquid ratio increases. It was also found that an increasing in the acid concentration always increases the dissolution mineral rate. It was determined that the dissolution rate fit well to the diffusive control model and the activation energy of this dissolution process was found to be 13 $\mathrm{KJ} / \mathrm{mol}$.
\end{abstract}




\section{INTRODUCCIÓN}

La hidroboracita es un borato de calcio y magnesio hexahidratado $\left(\mathrm{CaO} \cdot \mathrm{MgO} \cdot 3 \mathrm{~B}_{2} \mathrm{O}_{3} \cdot 6 \mathrm{H}_{2} \mathrm{O}\right)$. El borato se caracteriza porque es insoluble en agua fría y caliente, pero se disuelve en agua acidificada obteniéndose como producto principal, ácido bórico. La producción de ácido bórico ha incrementado recientemente debido al aumento de su uso en la industria del vidrio; esmaltes; productos químicos derivados como fluoboratos, floruros de boro, aleaciones de boro, carburo de boro, ferroboro, hidruro de boro, etc. Sus usos están relacionados, también, con la fruticultura en el lavado de citrus y conservación de los mismos; fungicidas; cosmética y farmacia; incectisidas; industria textil y con la preparación de cerámicas especiales, etc. (Flores, 2004). Los métodos tradicionales para la producción de ácido bórico a partir de minerales borados utilizan ácidos fuertes tales como ácido sulfúrico ó ácido clorhídrico. Sin embargo, estos métodos son antieconómicos debido a que la gran cantidad de impurezas alcalinas en el mineral lleva a un alto consumo del ácido, y a la baja importancia industrial de los subproductos que se obtienen de estas reacciones. En consecuencia, algunos autores estudiaron la disolución de boratos en otras soluciones ácidas, así por ejemplo: Kurtbaş et al. (2006) estudiaron la disolución de colemanita en soluciones acuosas de $\mathrm{SO}_{2}$ y ácido bórico. Determinaron que la velocidad de transformación del mineral es bien correlacionada por el modelo de Avrami. Demirkiran y Künkül (2007), investigaron la disolución de ulexita en soluciones de ácido perclórico y determinaron que la velocidad de disolución puede ser descripta, también, por el modelo de Avrami. Por otra parte Demirkiran (2009) estudió la cinética de disolución de ulexita en soluciones de nitrato de amonio. Determinó que, en ambos casos, la reacción ocurre con control químico. Ekmekyapar et al. (2008) estudiaron la cinética de disolución de ulexita en soluciones de ácido acético demostrando que la misma obedece al modelo del frente móvil. Tuba y Yartasi (2009) investigaron la cinética de reacción entre ulexita y ácido fosfórico demostrando que la velocidad de disolución es controlada por el proceso difusivo en la capa de producto que rodea al mineral. Kuşlu et al. (2010) investigaron la cinética de disolución de ulexita en soluciones de pentaborato con dióxido de carbono en un reactor batch. Observaron que los datos experimentales ajustan con el modelo del frente móvil y que el sistema es controlado por la reacción química. Por otra parte, Morales et al. (2010) estudiaron la disolución de hidroboracita en acido clorhídrico y determinaron que el proceso ocurre con control difusivo. Ekmekyapar et al. (2010) investigaron la cinética de disolución de ulexita en ácido cítrico en un reactor batch. Demostraron que la misma es controlada por la reacción química. En este trabajo se estudia la disolución de hidroboracita en soluciones de ácido nítrico $\left(\mathrm{HNO}_{3}\right)$. La importancia de este estudio reside en el hecho de que los subproductos que se obtienen de la reacción entre la hidroboracita y el $\mathrm{HNO}_{3}$, nitrato de calcio y nitrato de magnesio, tienen una gran importancia industrial debido a su uso como fertilizantes. Se investigan, simultáneamente, los efectos que tienen los factores temperatura, tamaño de partícula, relación sólido/líquido, y concentración de ácido sobre la velocidad de disolución del mineral. Se determina el modelo cinético y se evalúan los parámetros cinéticos: factor de frecuencia y energía de activación.

\section{PARTE EXPERIMENTAL}

Los experimentos de disolución de hidroboracita, se llevaron a cabo en un reactor de tipo tanque agitado discontinuo, de acero inoxidable, a escala laboratorio. La tapa del reactor posee un orificio central por donde se introduce el eje de un agitador mecánico, un orificio descentralizado, que se utiliza para cargar la muestra de mineral y un tubo soldado que se utiliza para tomar las muestras de la fase líquida. Durante la experimentación el reactor se introdujo en un baño termostático con temperatura controlada y se mantuvo el medio agitado mecánicamente. Se seleccionaron como factores (variables independientes) del proceso a los siguientes: $T$ (temperatura), $\mathrm{d}_{\mathrm{p}}$ (diámetro de partícula); $\mathrm{Ra}$ (relación masa de sólido a volumen de líquido) y $\mathrm{C}_{\mathrm{A}}$ (concentración del ácido). En la Tabla 1 se muestran los niveles de los $\mathrm{k}=4$ factores usados en los experimentos. Se uso un diseño factorial completo a dos niveles para cada parámetro (Küehl, 2003). Se realizó un análisis de experimentos para determinar qué factores afectan la transformación del reactivo sólido. Se consideraron los factores en forma independiente y las interacciones de los mismos, estableciéndose un intervalo de confianza del $95 \%$. Se pudo observar que los efectos significativos más importantes son los de las variables $d_{p}, C_{A},\left(d_{p} \times C_{A}\right)$ y 
$\mathrm{Ra}$, en orden decreciente. El efecto de la temperatura, $\mathrm{T}$, estaría en segundo orden de importancia y los efectos de las interacciones restantes no resultaron significativos.

Tabla 1: Factores y sus valores usados en los experimentos

\begin{tabular}{|c|c|c|c|c|}
\hline Niveles & $\begin{array}{c}\text { Tamaño de } \\
\text { partícula }(\mathrm{mm}) \\
\mathrm{d}_{\mathrm{p}}\end{array}$ & $\begin{array}{c}\text { Temperatura } \\
\left({ }^{\circ} \mathrm{C}\right) \\
\mathrm{T}\end{array}$ & $\begin{array}{c}\text { Sólido/líquido } \\
(\mathrm{g}(\mathrm{S}) / \mathrm{ml}(\mathrm{L})) \\
\mathrm{Ra}\end{array}$ & $\begin{array}{c}\text { Concentración } \\
\text { del ácido }(\mathrm{N}) \\
\mathrm{C}_{\mathrm{A}}\end{array}$ \\
\hline- & 0.326 & 60 & 0.01 & 0.23 \\
\hline+ & 0.774 & 70 & 0.02 & 0.50 \\
\hline
\end{tabular}

Se mantuvo la velocidad de agitación constante e igual a $520 \mathrm{rpm}$ después de verificar que la misma no tiene efectos significativos sobre la velocidad de disolución del mineral. La concentración en $\mathrm{B}_{2} \mathrm{O}_{3}$ del mineral utilizado en las experiencias fue $36.65 \%$. Se eligió como única respuesta del sistema al grado de transformación que experimenta el reactivo sólido, $\mathrm{X}$, con respecto de la masa de $\mathrm{B}_{2} \mathrm{O}_{3}$, y se la definió como:

$$
\mathrm{X}=\frac{\mathrm{M}_{\mathrm{B}}^{\circ}-\mathrm{M}_{\mathrm{B}}}{\mathrm{M}_{\mathrm{B}}^{\circ}}=\frac{\mathrm{M}_{\mathrm{B}(\mathrm{L})}}{\mathrm{M}_{\mathrm{B}}^{\mathrm{o}}}
$$

$\mathrm{M}_{\mathrm{B}}^{\circ}=$ masa inicial de $\mathrm{B}_{2} \mathrm{O}_{3}$ asociada a la partícula.

$\mathrm{M}_{\mathrm{B}}=$ masa asociada en un tiempo dado.

$\mathrm{M}_{\mathrm{B}(\mathrm{L})}=\mathrm{M}_{\mathrm{B}}^{\circ}-\mathrm{M}_{\mathrm{B}}=$ masa de $\mathrm{B}_{2} \mathrm{O}_{3}$ disuelta en la solución en un tiempo dado.

La definición de $X$ dada por la ec. (1), fue precisamente la que se utilizó para seguir el curso de la disolución. Para ello, a tiempos de reacción previamente programados se tomaron muestras de la mezcla reaccionante, las cuales fueron inmediatamente filtradas. Del líquido filtrado se tomó una alícuota y se la diluyó con agua destilada. La solución diluida se analizó mediante volumetría. Se utilizó hidróxido de sodio como disolución valorante del ácido nítrico, y a continuación se agregó manitol para valorar el ácido bórico con la misma disolución de hidróxido de sodio.

\section{RESULTADOS Y DISCUSIÓN}

La reacción química de la hidroboracita con $\mathrm{HNO}_{3}$ es:

$$
\begin{aligned}
& \mathrm{HNO}_{3}+\mathrm{H}_{2} \mathrm{O} \rightarrow \mathrm{NO}_{3(\mathrm{ac})}^{-}+\mathrm{H}_{3} \mathrm{O}_{(\mathrm{ac})}^{+} \\
& \begin{aligned}
\mathrm{CaO} . \mathrm{MgO} .3 \mathrm{~B}_{2} \mathrm{O}_{3} \cdot 6 \mathrm{H}_{2} \mathrm{O}_{(\mathrm{s})}+4 \mathrm{NO}_{3(\mathrm{ac})}^{-}+4 \mathrm{H}_{3} \mathrm{O}_{(\mathrm{ac})}^{+} \rightarrow & 6 \mathrm{H}_{3} \mathrm{BO}_{3(\mathrm{ac})}+\mathrm{Ca}\left(\mathrm{NO}_{3}\right)_{2(\mathrm{ac})}+ \\
+ & \mathrm{Mg}\left(\mathrm{NO}_{3}\right)_{2(\mathrm{ac})}+3 \mathrm{H}_{2} \mathrm{O}
\end{aligned}
\end{aligned}
$$

En la reacción (3) se forman nitrato de calcio y nitrato de magnesio que por su solubilidad permanecen en solución. Ambos productos tienen una gran importancia industrial dado que el nitrato de magnesio y el nitrato de calcio se utilizan para el aporte de Ca y $\mathrm{Mg}$ al suelo; y debido a su alta solubilidad, tanto el $\mathrm{N}$ como el $\mathrm{Ca}$ y el $\mathrm{Mg}$ pasan en forma inmediata al mismo.

En la Fig. 1 se presenta la influencia del tamaño de partícula sobre la disolución de hidroboracita en $\mathrm{HNO}_{3}(0.23 \mathrm{~N})$ a $60{ }^{\circ} \mathrm{C}$ y con la relación sólido/líquido $0.02 \mathrm{~g}(\mathrm{~S}) / \mathrm{ml}(\mathrm{L})$. Se observa que la velocidad de disolución de la hidroboracita en $\mathrm{HNO}_{3}$ aumenta con la disminución del tamaño de partícula del mineral, así por ejemplo la disminución de casi el doble en el tamaño de la partícula del mineral hace incrementar en un $18 \%$ el grado de disolución del mismo, a los 120 segundos de comenzada la disolución. Un efecto similar se manifiesta a otras temperaturas de disolución, a otras relaciones sólido líquido y a otra concentraciones de ácido. 


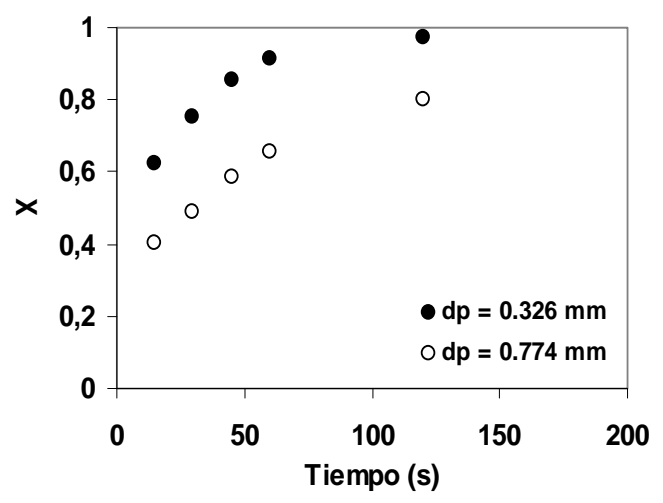

Fig. 1: Influencia del diámetro de partícula sobre la velocidad de disolución

Por otra parte, en la Fig. 2 se presentan los resultados experimentales de la disolución de hidroboracita en $\mathrm{HNO}_{3}(0.23 \mathrm{~N})$ a $50^{\circ} \mathrm{C}, 60^{\circ} \mathrm{C}$ y $70^{\circ} \mathrm{C}$, para un diámetro de partícula de $0.774 \mathrm{~mm}$ y una relación sólido/ líquido $0.02 \mathrm{~g}(\mathrm{~S}) / \mathrm{ml}(\mathrm{L})$. Se observa que a medida que aumenta la temperatura aumenta la velocidad de disolución del mineral. Así para un incremento de $10^{\circ} \mathrm{C}$ en la temperatura (de $60^{\circ} \mathrm{C}$ a $70^{\circ} \mathrm{C}$ ), el grado de disociación del mineral se incrementa en un $20 \%$ a los 60 segundos de comenzada la disolución.

Se realizaron, también, experimentos para investigar el efecto de la relación sólido/líquido en la disolución de hidroboracita en $\mathrm{HNO}_{3}$. En la Fig. 3 se presentan datos para un diámetro de partícula de $0.774 \mathrm{~mm}$, a $60^{\circ} \mathrm{C}$ y una concentración de ácido $0.23 \mathrm{~N}$. Se observa que un aumento en la relación sólido/líquido hace disminuir el grado de transformación del mineral y que un incremento en la relación sólido/líquido del doble, hizo disminuir el grado de transformación del mineral en un $30 \%$ a los 60 segundos de comenzada la disolución.

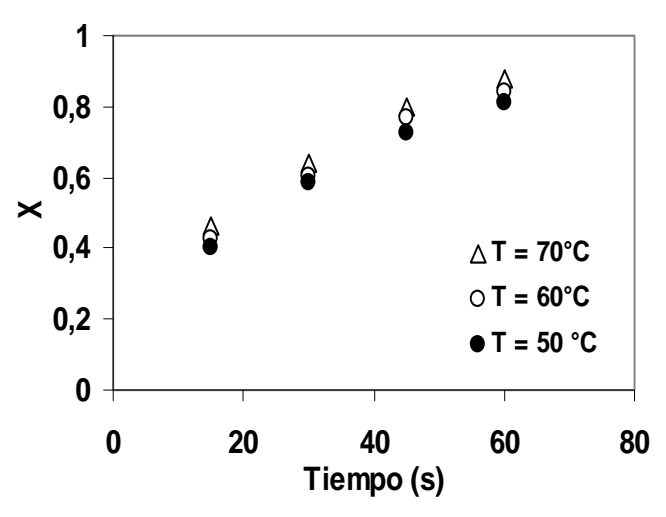

Fig. 2: Influencia de la temperatura sobre la velocidad de disolución

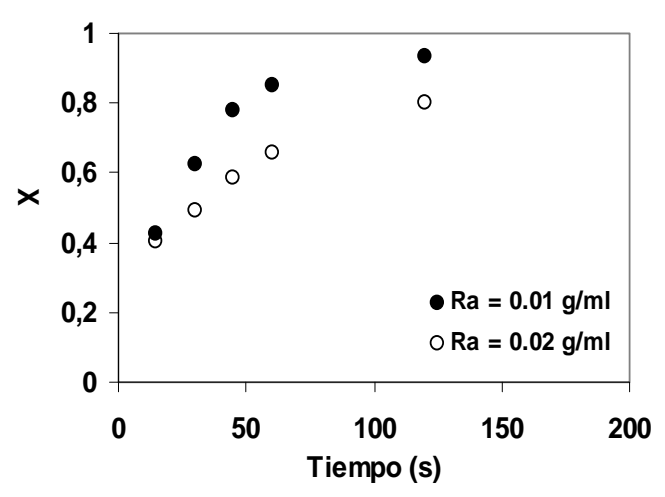

Fig. 3: Influencia de la relación sólido/líquido sobre la velocidad de disolución

Con respecto a la influencia de la concentración del ácido sobre la velocidad de disolución, como es de esperar para este tipo de sistemas, el grado de transformación del mineral aumenta siempre con el aumento de la concentración del ácido.

\section{MODELADO CINÉTICO}

La velocidad de reacción entre un sólido y un fluido puede ser expresada por modelos de reacción heterogéneos y homogéneos. El modelo heterogéneo del frente móvil, considera que la reacción tiene lugar en la superficie externa de la partícula reactiva. Cuando no se forman cenizas, como en la ec. (3), el sólido va disminuyendo de tamaño hasta que desaparece. Para una reacción de esta clase, se considera que ocurren las siguientes etapas en serie (Levenspiel, 1972): difusión del reactante en la película de fluido que rodea al sólido, hasta la superficie del mismo; reacción química sobre la superficie, entre el fluido reactante y el sólido; y difusión de los productos de 
reacción, desde la superficie del sólido, a través de la película líquida que rodea al mismo. Si el proceso es controlado por la resistencia en la película del fluido, el grado de transformación del reactivo sólido puede ser escrito como:

$$
X=\frac{6 b k_{g} C_{A}}{\rho_{B} d_{p}} t
$$

$\mathrm{b}$ = coeficiente estequiométrico del sólido en la ec. (3).

$\mathrm{k}_{\mathrm{g}}=$ coeficiente de transferencia de masa.

$\rho_{B}=$ densidad del reactivo sólido.

Si el proceso es controlado por la resistencia de la reacción química superficial, el grado de transformación del reactivo sólido puede ser escrito como:

$$
1-(1-X)^{1 / 3}=\frac{2 b k_{s} C_{A}}{\rho_{B} d_{p}} t
$$

Además de los modelos heterogéneos se pueden usar modelos pseudo-homogéneos para derivar ecuaciones de velocidad de reacción heterogéneas. La ec. (6) corresponde a un modelo de pseudo-homogéneo de primer orden y la ec. (7) a un modelo pseudo-homogéneo de segundo orden.

$$
\begin{aligned}
& -\ln (1-X)=b_{1} t \\
& (1-X)^{-1}-1=b_{1} t
\end{aligned}
$$

En adición a estos modelos pseudo-homogéneos, se puede usar el modelo de Avrami (Avrami, 1939), que es un modelo de control difusional:

$$
-\ln (1-X)=b_{1} t^{b_{2}}
$$

Para el tratamiento cinético de los datos experimentales se desarrolló un software específico que permitió correlacionar los datos cinéticos mediante modelos presentados, ec. (4) a ec. (8), para sistemas fluido-sólido reactivo; habiéndose encontrado que el único modelo capaz de ajustar los datos con un 95\% de certeza, es el modelo de Avrami. Reemplazando en la ec. (8) el valor de los parámetros encontrados resulta:

$$
-\ln (1-X)=80.42 \exp \left(\frac{-1598}{T}\right) \frac{C_{A}^{0.58}}{d_{p}^{0.79} \mathrm{Ra}^{0,36}} t^{0.8}
$$

$$
\text { E: energía de activación }=13 \mathrm{~kJ} / \mathrm{mol} \quad \mathrm{y} \quad \mathrm{b}_{2}=0.8
$$

El valor de la energía de activación en una reacción de disolución puede ser usado para predecir la etapa controlante de velocidad. Es decir, un proceso controlado por difusión tiene un valor típico de la energía de activación entre 4 y $12 \mathrm{KJ} / \mathrm{mol}$, mientras que un proceso controlado químicamente tiene un valor de la energía de activación mayor que $40 \mathrm{KJ} / \mathrm{mol}$ (Demirkiran, 2009). Por otra parte, los procesos heterogéneos controlados por difusión son ligeramente dependientes de la temperatura, mientras que los procesos controlados químicamente son fuertemente dependientes de la temperatura. Por lo tanto, dado que el proceso aquí presentado depende muy poco de la temperatura y que el valor de la energía de activación es de $13 \mathrm{~kJ} / \mathrm{mol}$ se concluye que el proceso de disolución de hidroboracita en soluciones de $\mathrm{HNO}_{3}$ es controlado por mecanismos difusivos. En la Fig. 4 se presenta el ajuste entre los valores de conversión experimental $\left(\mathrm{X}_{\mathrm{ex}}\right)$ y los valores predichos por el modelo cinético $\left(\mathrm{X}_{\mathrm{m}}\right)$. Puede observase que el ajuste es bastante bueno. 


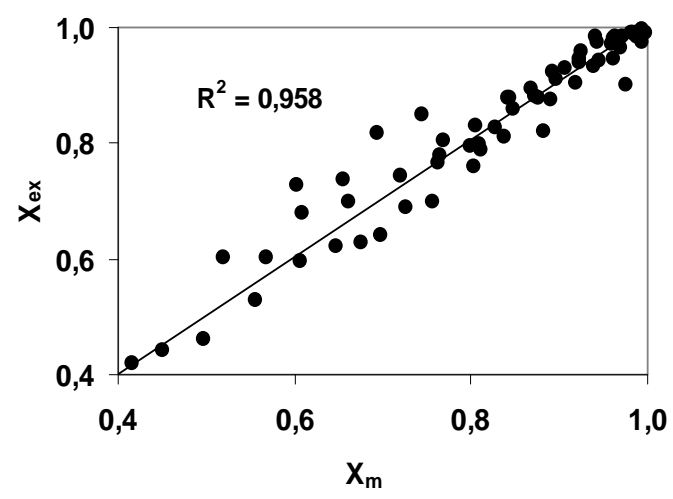

Fig. 4: Ajuste entre los valores de conversión experimental y los valores predichos por el Modelo

\section{CONCLUSIONES}

Se observa que la velocidad de disolución de hidroboracita en soluciones de $\mathrm{HNO}_{3}$ es bastante rápida, disminuye a medida que aumenta la relación sólido/líquido y aumenta con el aumento de la concentración de la solución de $\mathrm{HNO}_{3}$, con la disminución del tamaño de partícula y con el incremento de la temperatura; siendo este efecto el menos significativo. Dado que la energía de activación aparente es del orden de $13 \mathrm{~kJ} / \mathrm{mol}$ y que el proceso depende muy poco de la temperatura, es posible inferir que la etapa controlante del proceso es la de difusión del soluto o del solvente a través de los poros del sólido. Estas observaciones apoyan el modelo teórico encontrado, que es un modelo de control difusional. Se puede concluir, entonces, que la disolución de hidroboracita en soluciones de ácido nítrico es controlada por mecanismos difusivos.

\section{REFERENCIAS}

Avrami M., Kinetic of phase change I. General Theory, J. Chem. Phys., 7, 1103-1112 (1939).

Demirkiran N. y A. Künkül, Dissolution kinetics of ulexite in perchloric acid solutions, Int. J. Miner. Process, 83, 76-80 (2007).

Demirkiran N., Dissolution kinetics of ulexite in ammonium nitrate solutions, Hydrometallurgy, 95, 198-202 (2009).

Ekmekyapar A., N. Demirkiran y A. Künkül, Dissolution kinetics of ulexite in acetic acid solutions, Chemical Engineering Research and Design, 86, 1101-1016 (2008).

Ekmekyapar A , A. Künkül y N. Demirkiran, Kinetic investigation of reaction between mineral ulexite and citric acid, Mineral Processing and Extractive Metallurgy Review: An Internacional Journal, 31, 4, 250-255 (2010).

Flores, H. R., El Beneficio de los Boratos, Crisol Ediciones, Salta (2004).

Küehl R. O., Diseño de experimentos: Principios estadísticos de diseño y análisis de investigación, 1 ra edición, Ed. Thomson, México (2003).

Kurtbaş A., M.M. Kocakerim, O. Küçük y A. Yartaşi, Dissolution of colemanite in aqueous solutions saturated with both sulfur dioxide and boric acid, Ind. Eng. Chem Res., 45, 1857-1862 (2006).

Kuşlu S., F. Çavus Dişli y S. Çolak, Leaching kinetic of ulexite in borax pentahydrate solutions saturared with carbon dioxide, Journal of Industrial and Engineering Chemistry, 16, 673-679(2010).

Levenspiel O., Chemical Reaction Engineering, John Wiley \& Sons, New York (1972).

Morales G.V., O.D. Quiroga y E.N. Tolaba, Modelado cinético de la disolución de hidroboracita en ácido clorhídrico, Información Tecnológica, 21, 21-26 (2010).

Tuba Doğan H. y A. Yartaşi, Kinetic investigation of reaction between ulexite ore and phosphoric acid, Hydrometallurgy, Vol. 96, 294-299 (2009). 